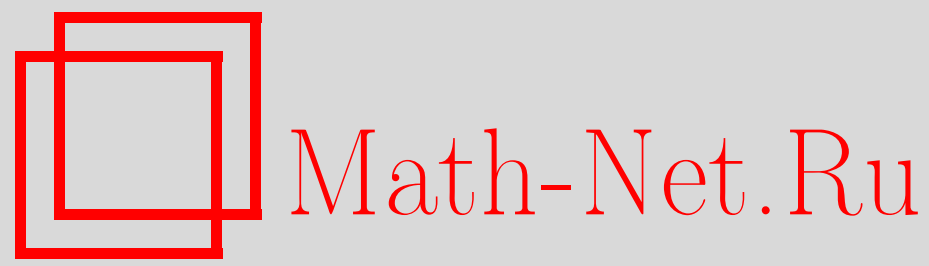

В. И. Масол, Асимптотика числа некоторых $k$ мерных подпространств над конечным полем, $\mathrm{Ma}$ тем. заметки, 1996, том 59, выпуск 5, 729-736

DOI: https://doi.org/10.4213/mzm1767

Использование Общероссийского математического портала MathNet.Ru подразумевает, что вы прочитали и согласны с пользовательским соглашением

http://www. mathnet.ru/rus/agreement

Параметры загрузки:

IP : 54.209 .52 .79

26 апреля 2023 г., 13:31:16

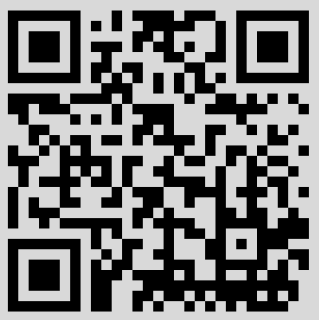




\section{АСИМПТОТИКА ЧИСЛА НЕКОТОРЫХ $k$-МЕРНЫХ ПОДПРОСТРАНСТВ НАД КОНЕЧНЫМ ПОЛЕМ}

\section{В.И. Масол}

Известно, что общее число $\left[\begin{array}{l}n \\ k\end{array}\right]$ различных $k$-мерных подпространств $V_{k, n} n$-мерного векторного пространства $V_{n}$ над конечным полем $\operatorname{GF}(q)$, содержашем $q$ элементов ( $q$ - степень простого числа), равно

$$
\left[\begin{array}{l}
n \\
k
\end{array}\right]=\prod_{i=0}^{k-1} \frac{q^{n-i}-1}{q^{k-i}-1}
$$

где $1 \leqslant k \leqslant n$ (см., например, [1, с. 219]). Примем, что $\left[\begin{array}{c}n \\ 0\end{array}\right]=1, n \geqslant 0$. Весом вектора $v \in V_{n}$ назовем число отличных от нуля компонент вектора $v$. Весом $k$-мерного подпространства $V_{k, n}$ пространства $V_{n}$ назовем число, равное минимальному весу вектора $v \in V_{k, n}$, отличного от нулевого, $1 \leqslant k \leqslant n$. В дальнейшем запись $V_{(k, n \mid \omega)}$ будет обозначать класс всех отличающихся между собой $k$-мерных подпространств пространства $V_{n}$, каждое из которых имеет вес $\omega$. Для мошности класса $V_{(k, n \mid \omega)}$ примем запись $\left[\begin{array}{c|c}n \\ k\end{array} \mid \omega\right]$. Очевидно, что

$$
\left[\begin{array}{l}
n \\
k
\end{array}\right]=\sum_{\omega \geqslant 1}\left[\begin{array}{l}
n \\
k
\end{array} \mid \omega\right],
$$

где $k>0$. Нас интересует вклад слагаемого $\left[\begin{array}{l|l}n \\ k\end{array} \mid 1\right]$ в общую сумму в правой части равенства (1) при $n \rightarrow \infty$. Всюду далее параметр $m$ равен $m=n-k$, причем $m \geqslant 0$.

Теорема 1. Для иелых $n \geqslant k \geqslant 1$

$$
\begin{aligned}
& {\left[\begin{array}{l|l}
n & 1 \\
k & 1
\end{array}\right]=\sum_{\mu=1}^{k}\left(q^{m}-1\right)^{\mu-1}\left(\left[\begin{array}{l}
n-\mu \\
k-\mu
\end{array}\right]+\left[\begin{array}{c|c}
n-\mu \\
k-\mu+1
\end{array}\right]\right),} \\
& {\left[\begin{array}{l|l}
n & 1 \\
k & 1
\end{array}\right]=\sum_{\nu=1}^{m+1}\left(\left[\begin{array}{l}
n-\nu \\
k-1
\end{array}\right]+\left[\begin{array}{l}
n-\nu \\
k-1
\end{array}\right] 1\left(^{m+1-\nu}-1\right)\right),}
\end{aligned}
$$


əде $\left[\begin{array}{l}d \\ p\end{array} \mid 1\right]=0$, если либо $p=0$, либо $p>d$.

Доказательству теоремы 1 предпошлем формулировку известного (см., например, [1, с. 219]) алгоритма построения $k \times n$ матрицы $B$, строки которой являются базисными векторами некоторого подпространства $V_{k, n} \subseteq V_{n}$.

Алгоритм. Выбираем $k$-подмножество $\left\{r_{1}, \ldots, r_{k}\right\}, 1 \leqslant r_{1}<\cdots<$ $r_{k} \leqslant n$, множества $\{1, \ldots, n\}$. В матрице $B$ образуем единичную $k \times k$ матрицу из столбцов с номерами из $\left\{r_{1}, \ldots, r_{k}\right\}$. В $i$-й строке матрицы $B$ записьваем нуль во все позиции $j>r_{i}, i=\overline{1, k}$. Оставшиеся места матрицы $B$ заполняем элементами поля независимым образом.

ДоКАЗАТЕЛЬСТВо тЕоремы 1. Анализ алгоритма позволяет заключить, что порождаемая им матрища $B$, строки которой образуют базис некоторого подпространства из класса $V_{(k, n \mid 1)}$, может быть одного и только одного из следующих трех видов: a) $n$-й столбец матрищы $B$ состоит из нулей, а оставшиеся $(n-1)$-мерные строки являются базисными для некоторого подпространства из класса $V_{(k, n-1 \mid 1)}$; б) $k$-я строка матрицы $B$ содержит, по крайней мере, два отличных от нуля элемента поля, причем один из них равен единице и расположен на $n$-й позиции, а остальные строки - базисные векторы некоторого подпространства из класса $V_{(k-1, n-1 \mid 1)}$; в) на позиции с номером $(k, n)$ в матрице $B$ находится единища, остальные элементы $k$-й строки равны нулю, а строки $(k-1) \times(n-1)$ подматрищы матрицы $B$ порождают некоторое $(k-1)$-мерное подпространство пространства $V_{n-1}$.

Нетрудно проверить, что число подпространств, удовлетворяющих а), равно $\left[\begin{array}{c|c}n-1 & 1\end{array}\right]$, б) - $\left[\begin{array}{c|c}n-1 \\ k-1 & 1\end{array}\right]\left(q^{m}-1\right)$, в $)-\left[\begin{array}{c}n-1 \\ k-1\end{array}\right]$. Суммируя числа, найденные в а), б) и в), получаем

$$
\left[\begin{array}{l|l}
n & 1 \\
k & 1
\end{array}\right]=\left[\begin{array}{c|c}
n-1 & 1 \\
k & 1
\end{array}\right]+\left[\begin{array}{c}
n-1 \\
k-1
\end{array}\right]+\left[\begin{array}{c|c}
n-1 & 1 \\
k-1 & 1
\end{array}\right]\left(q^{m}-1\right) .
$$

Применяя (4), находим для $m \geqslant 1$

$$
\begin{aligned}
& {\left[\begin{array}{l|l}
n & 1 \\
k & 1
\end{array}\right]=\left[\begin{array}{c|c}
n-1 & 1 \\
k & 1
\end{array}\right]+\left[\begin{array}{c}
n-1 \\
k-1
\end{array}\right]+\left(q^{m}-1\right)} \\
& \times\left(\left[\begin{array}{l|l}
n-2 & 1 \\
k-1 & 1
\end{array}\right]+\left[\begin{array}{l}
n-2 \\
k-2
\end{array}\right]+\left(q^{m}-1\right)\left[\begin{array}{l|l}
n-2 & 1 \\
k-2 & 1
\end{array}\right]\right)=\cdots \\
& =\sum_{\mu=1}^{k}\left(\left[\begin{array}{c|c}
n-\mu & 1 \\
k-\mu+1 & 1
\end{array}\right]+\left[\begin{array}{c}
n-\mu \\
k-\mu
\end{array}\right]\right)\left(q^{m}-1\right)^{\mu-1} \text {. }
\end{aligned}
$$

Равенства (5) и $\left[\begin{array}{c|c}n & 1 \\ n & 1\end{array}\right]=1$ доказывают (2). Аналогичным образом, с помощью (4) можно проверить (3). Теорема 1 доказана.

Обозначим

$$
P(n, k)=\left[\begin{array}{l|l}
n & 1 \\
k & 1
\end{array}\right] /\left[\begin{array}{l}
n \\
k
\end{array}\right], \quad 1 \leqslant k \leqslant n .
$$


TEOPEMA 2. Ecлu

$$
m=o(\ln k), \quad n \rightarrow \infty,
$$

mo

$$
P(n, k) \rightarrow 1, \quad n \rightarrow \infty
$$

если при $n \rightarrow \infty$

$$
k=x q^{m}(1+o(1)),
$$

где $x$ - фиксированное положительное число, то

$$
P(n, k) \rightarrow 1-e^{-x}, \quad n \rightarrow \infty ;
$$

если

$$
k=o\left(q^{m}\right), \quad n \rightarrow \infty
$$

mo

$$
P(n, k) \rightarrow 0, \quad n \rightarrow \infty .
$$

ЗАмечАниЕ. Теорема 2 может быть проинтерпретирована следующим образом. Если $n$ и $k$ незначительно отличаются между собой, например, на величину, не превосходящую $o(\ln k)$, т.е. $n-k \ll \ln k$, то асимптотически при $n \rightarrow \infty$ все $k$-мерные подпространства $n$-мерного пространства над полем $\mathrm{GF}(q)$ обладают весом, равньм 1 . Если $n$ отличается от $k$ на величину порядка $\ln k$, то имеет место экспоненциальное поведение числа $\left[\begin{array}{l|l}n \\ k\end{array} 1\right.$ 1 $\left[\begin{array}{l}n \\ k\end{array}\right]^{-1}$ при $n \rightarrow \infty$. Наконец, если $n \gg k+\ln k$, то асимптотически при $n \rightarrow \infty$ все $k$-мерные подпространства $n$-мерного пространства над $\mathrm{GF}(q)$ обладают весом, большим или равным 2 .

Доказательству теоремы 2 предпошлем следующие леммы.

ЛЕмма 1. Пусть $0 \leqslant k \leqslant n ;$ тогда

$$
\left[\begin{array}{l}
n \\
k
\end{array}\right]=q^{m k} \prod_{s=1}^{m}\left(q^{s}-q^{-k}\right)\left(q^{s}-1\right)^{-1} .
$$

ДоКАЗАТЕЛЬСТВО этой леммы можно найти, например, в [1, с. 49].

Лемма 2. Для целых неотрицательных $k$ ut

$$
q^{k} \sum_{s=1}^{t}\left(q^{k+s}-1\right)^{-1} \leqslant\left(1-q^{-t}\right)(q-1)^{-1}\left(1-q^{-k-1}\right)^{-1} .
$$

ДокАЗАТЕЛЬСТвО. Достаточно заметить, что

$$
q^{k} \sum_{s=1}^{t}\left(q^{k+s}-1\right)^{-1} \leqslant\left(q-q^{-k}\right)^{-1} \sum_{s=1}^{t} q^{-s+1}
$$

и воспользоваться формулой суммы членов конечной геометрической прогрессии. 
ЛЕмма 3. Для иелых неотричательных $s$ ut

$$
\left[\begin{array}{l|l}
t+s & 1 \\
s+1 & 1
\end{array}\right] \leqslant(t+s) q^{t s}
$$

ДокаЗАтеЛЬСтво. Если $t=0$, то (14) очевидно. Пусть $t \geqslant 1$. Обозначим через $Q$ левую часть неравенства (14). Покажем, что для $s-t \geqslant 1$

$$
Q<q^{t s}\left(\frac{e}{q}\right)^{2}<(t+s) q^{t s}
$$

Поскольку $Q \leqslant\left[\begin{array}{c}t+s \\ s+1\end{array}\right]$, то используя $(12)$, находим

$$
Q \leqslant q^{(t-1)(s+1)} \prod_{\rho=1}^{t-1}\left(1+\frac{1-q^{-s-1}}{q^{\rho}-1}\right) .
$$

Отсюда получаем в силу условия $s-t \geqslant 1$ и оценки $1+z<e^{z}, z \neq 0$,

$$
Q<q^{t s-2} \exp \left\{\sum_{\rho=1}^{t-1}\left(q^{\rho}-1\right)^{-1}\right\} .
$$

Применяя к правой части последнего неравенства соотношение (13), имеем

$$
Q<q^{t s-2} \exp \left\{q(q-1)^{-2}\left(1-q^{-t+1}\right)\right\},
$$

и так как $q(q-1)^{-2}\left(1-q^{-t+1}\right)<2$, то $Q<q^{t s}(e / q)^{2}$. Для завершения доказательства (15) достаточно заметить, что при $t \geqslant 1$ и $s-t \geqslant 1$ $(e / q)^{2}<t+s$.

Покажем, что (14) выполняется при $t \geqslant s$. Для $t \in\{1,2,3\}$ неравенство (14) легко проверить с помощью соотношения (4) и известного равенства $\left[\begin{array}{l}n \\ k\end{array}\right]=\left[\begin{array}{l}n-1 \\ k-1\end{array}\right]+q^{k}\left[\begin{array}{c}n-1 \\ k\end{array}\right]$ (см., например, [1, с. 49]). Для $t \geqslant 4$ и $s \in\{0,1\}$ оценка (14) следует из того, что $\left[\begin{array}{l|l}t & 1 \\ 1 & 1\end{array}\right]=t$ и с учетом (3)

$$
\left[\begin{array}{c|c}
t+1 & 1 \\
2 & 1
\end{array}\right]=(q-1)^{-1}(t+1)\left(q^{t}-2^{-1} t(q-1)-1\right) \leqslant(t+1) q^{t} .
$$

Пусть $t \geqslant 4, s \geqslant 2$ и $t \geqslant s$. Для доказательства (14) воспользуемся методом математической индукции по параметру $s \geqslant 1$. Вьше была установлена оценка (14) для $s=1$. Индукционньй шаг от $s-1$ к $s$ можно обосновать следуюшим образом. В силу (3) получаем

$$
Q=\sum_{\nu=1}^{t}\left(\left[\begin{array}{c}
t+s-\nu \\
s
\end{array}\right]+\left(q^{t-\nu}-1\right)\left[\begin{array}{c|c}
t+s-\nu & 1 \\
s &
\end{array}\right]\right)
$$


Используя соотношения $(15),(16)$ и предположение индукции, находим

$$
Q \leqslant \sum_{\nu=1}^{t}\left(\left[\begin{array}{c}
t+s-\nu \\
s
\end{array}\right]+(t+s-\nu) q^{s(t+1-\nu)-1}\right) .
$$

Нетрудно проверить неравенство

$$
\sum_{\nu=1}^{t}(t+s-\nu) q^{s(t+1-\nu)-1} \leqslant(t+s-1) q^{s(t+1)-1}\left(q^{s}-1\right)^{-1} .
$$

Далее покажем, что

$$
\sum_{\nu=1}^{t}\left[\begin{array}{c}
t+s-\nu \\
s
\end{array}\right]<q^{t s} e^{2}\left(q^{s}-1\right)^{-1} .
$$

Действительно, с помошью (12) и (13) получаем, что

$$
\sum_{\nu=1}^{t}\left[\begin{array}{c}
t+s-\nu \\
s
\end{array}\right] \leqslant q^{t s} \sum_{\nu=1}^{t} q^{-s \nu} \exp \left\{\left(1-q^{-s}\right) q\left(1-q^{-t+\nu}\right)(q-1)^{-2}\right\},
$$

но, поскольку $\left(1-q^{-s}\right) q\left(1-q^{-t+\nu}\right)(q-1)^{-2}<2$, то

$$
\sum_{\nu=1}^{t}\left[\begin{array}{c}
t+s-\nu \\
s
\end{array}\right]<q^{t s} e^{2} \sum_{\nu=1}^{t} q^{-s \nu}
$$

отсюда непосредственно следует (19).

Оценки (17)-(19) позволяют заключить, что

$$
Q<q^{t s}\left((t+s) q^{s-1}\left(q^{s}-1\right)^{-1}-q^{-1}+\left(e^{2}-q^{-1}\right)\left(q^{s}-1\right)^{-1}\right) .
$$

Несложно убедиться, что правая часть соотношения (20) не превосходит $(t+s) q^{t s}$ для $t \geqslant 4$ и $s \geqslant 2$. Лемма 3 доказана.

ДОКАЗАТЕЛЬСТВО ТЕОРЕМЫ 2. С ПОМОщЬю (2) находим для $m \geqslant 1$

$$
P(n, k)=S_{1}+S_{2}+S_{3},
$$

где

$$
\begin{aligned}
S_{1} & =\sum_{\mu=1}^{k-m}\left[\begin{array}{c}
n-\mu \\
k-\mu+1
\end{array}\right]\left(q^{m}-1\right)^{\mu-1} /\left[\begin{array}{l}
n \\
k
\end{array}\right], \\
S_{2} & =\sum_{\mu=1}^{k-m}\left[\begin{array}{c}
n-\mu \\
k-\mu
\end{array}\right]\left(q^{m}-1\right)^{\mu-1} /\left[\begin{array}{l}
n \\
k
\end{array}\right], \\
S_{3} & =\sum_{\nu=0}^{m-1}\left(\left[\begin{array}{c}
m+\nu \\
\nu
\end{array}\right]+\left[\begin{array}{c}
m+\nu \\
\nu+1
\end{array}\right]\right)\left(q^{m}-1\right)^{k-\nu-1} /\left[\begin{array}{l}
n \\
k
\end{array}\right] .
\end{aligned}
$$


Используя лемму 1 , получаем

$$
S_{1} \leqslant q^{-k} \sum_{\mu=1}^{k-m}\left(q-q^{-m+1}\right)^{\mu-1}
$$

с помошью (12) устанавливаем неравенство

$$
S_{2} \leqslant 1-\left(1-q^{-m}\right)^{k-m}
$$

применяя лемму 3 , приходим к оценке

$$
S_{3} \leqslant \sum_{\nu=0}^{m-1}\left((m+\nu) q^{m \nu}+\left[\begin{array}{c}
m+\nu \\
\nu
\end{array}\right]\right)\left(q^{m}-1\right)^{k-\nu-1} /\left[\begin{array}{l}
n \\
k
\end{array}\right]
$$

откуда с учетом леммы 1 следует, что

$$
S_{3} \leqslant\left(1-q^{-m}\right)^{k-1} \sum_{\nu=0}^{m-1}(m+\nu+1) q^{m(\nu-1)}\left(q^{m}-1\right)^{-\nu} .
$$

Условие (10), равенство (21) и оценки (22)-(24) приводят к (11). Условие $(8)$, равенство (21) и (22)-(24) дают при $n \rightarrow \infty$

$$
P(n, k) \leqslant 1-e^{-x} .
$$

Покажем, что в условиях теоремы 2

$$
\begin{aligned}
P(n, k) \geqslant & 1-\left(1-q^{-m}\right)^{k}-\left(1-q^{-m}\right)(q-1)^{-1}\left(q^{k+1}-1\right)^{-1} \\
& \times q^{2-m} \sum_{\mu=1}^{k}\left(q-q^{-m+1}\right)^{\mu-1} .
\end{aligned}
$$

Действительно, соотношения (2) и (12) дают

$$
\begin{aligned}
P(n, k) & \geqslant\left[\begin{array}{l}
n \\
k
\end{array}\right]^{-1} \sum_{\mu=1}^{k}\left[\begin{array}{l}
n-\mu \\
k-\mu
\end{array}\right]\left(q^{m}-1\right)^{\mu-1} \\
& =q^{-m} \sum_{\mu=1}^{k}\left(1-q^{-m}\right)^{\mu-1} \prod_{s=1}^{m}\left(1-\left(q^{\mu}-1\right)\left(q^{k+s}-1\right)^{-1}\right) .
\end{aligned}
$$

Соотношение (27) и неравенство

$$
\prod_{i=1}^{m}\left(1-\gamma_{i}\right) \geqslant 1-\sum_{i=1}^{m} \gamma_{i}
$$


где $0<\gamma_{i}<1, i=\overline{1, m}$, позволяют перейти к оценке

$$
P(n, k) \geqslant 1-\left(1-q^{-m}\right)^{k}-q^{-m+1} \sum_{s=1}^{m}\left(q^{k+s}-1\right)^{-1} \sum_{\mu=1}^{k}\left(q-q^{-m}\right)^{\mu-1} .
$$

С помощью (13) и (28) получаем

$$
\begin{aligned}
& P(n, k) \geqslant 1-\left(1-q^{-m}\right)^{k} \\
& \quad-\left(1-q^{-m}\right)(q-1)^{-1}\left(1-q^{-k-1}\right)^{-1} q^{-k-m+1} \sum_{\mu=1}^{k}\left(q-q^{-m}\right)^{\mu-1}
\end{aligned}
$$

что равносильно, очевидно, (26).

Из неравенств $(26)$ и $1 \geqslant P(n, k)$ следует, что при выполнении условия (6) имеет место (7). Используя (25) и (26), убеждаемся (с учетом условия (8)) в справедливости (9). Теорема 2 доказана.

Tеорема 3. Условие

$$
\frac{q^{m}-(q-1) m-1}{q-1}<k
$$

где $1 \leqslant k \leqslant n$, является необходимым $и$ достаточным для того, чтобы выполнялось равенство

$$
\left[\begin{array}{l}
n \\
k
\end{array}\right]=\left[\begin{array}{l|l}
n & 1 \\
k &
\end{array}\right]+\left[\begin{array}{l|l}
n & 2 \\
k &
\end{array}\right]
$$

Доказательству теоремы 3 предпошлем следующие леммы.

ЛЕмма 4. Пусть строки $k \times m(k \geqslant 1, m \geqslant 2)$ матрици $B^{\prime}$, состоящей из әлементов поля $\mathrm{GF}(q)$, удовлетворяют условиям:

(у1) каждая строка матрииы $B^{\prime}$ имеет вес $\omega \geqslant 2$;

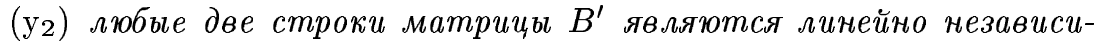
мымми над полем $\mathrm{GF}(q)$.

Тогда $k \leqslant\left(q^{m}-(q-1) m-1\right) /(q-1)$.

ДокАЗАТЕЛЬСТво. Максимальное число отличающихся между собой $m$-мерных строк, каждая из которых имеет вес $\omega \geqslant 2$, равно, очевидно, $q^{m}-(q-1) m-1$. Нетрудно видеть, что среди указанных $q^{m}-(q-1) m-1$ строк максимальное число строк, удовлетворяющих условию $\left(\mathrm{y}_{2}\right)$, равно $\left(q^{m}-(q-1) m-1\right) /(q-1)$. Лемма 4 доказана.

ЛЕмма 5. Пусть $m \geqslant 2 u$

$$
1 \leqslant k \leqslant \frac{q^{m}-(q-1) m-1}{q-1} .
$$

Тогда существует число $\omega, \omega \geqslant 3$, такое, что $V_{(k, n \mid \omega)} \neq \varnothing$. 
ДоКАЗАТЕЛЬСТВо. Рассмотрим $k \times n$ матрицу $B$, в которой столбцы с номерами $m+1, m+2, \ldots, n$ образуют единичную $k \times k$ матрицу, а столбцы с номерами $1,2, \ldots, m$ образуют матрицу, которую обозначим $B^{\prime}$. Строки матрицы $B^{\prime}$ будем заполнять таким образом, чтобы выполня-

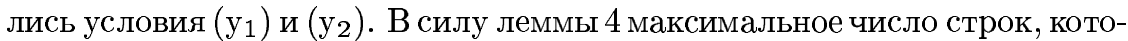
рое можно заполнить указанным образом, равно $\left(q^{m}-(q-1) m-1\right) /(q-1)$. Отсюда с учетом (31) получаем, что все строки матрицы $B^{\prime}$ могут удовле-

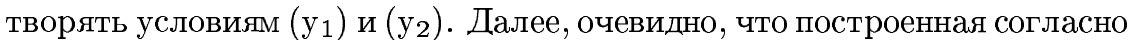
алгоритму $k \times n$ матрица $B$ c $k \times m$ матрищей $B^{\prime}, m$-мерные строки которой удовлетворяют условиям $\left(\mathrm{y}_{1}\right)$ и $\left(\mathrm{y}_{2}\right)$, состоит из $n$-мерных базисных векторов одного из подпространств, принадлежашего классу $V_{(k, n \mid \omega)}$ для некоторого $\omega \geqslant 3$. Лемма 5 доказана.

ДОКАЗАТЕЛЬСТВО ТЕОРЕМЫ 3 . Для $m \in\{0,1\}$ теорему 3 легко установить с помощью алгоритма. Для $m \geqslant 2$ обоснование необходимости (достаточности) условия (29) нетрудно осуществить, используя лемму 5 (лемму 4). Теорема 3 доказана.

Теоремы 2 и 3 позволяют для некоторых значений $k$ получить оценку величины $\left[\begin{array}{c|c}n \\ k\end{array} \mid 2\right]$, а именно, справедлива

Теорема 4. Если выполняется условие (6), то при $n \rightarrow \infty$ для $\omega \in\{2,3, \ldots, n\} \quad\left[\begin{array}{c}n \\ k\end{array} \mid \omega\right] /\left[\begin{array}{l}n \\ k\end{array}\right] \rightarrow 0 ;$ если $k=y\left(q^{m}-(q-1) m\right)$, где $y-$ фиксированное число, $(q-1)^{-1} \leqslant y<\infty$, то при $n \rightarrow \infty$

$$
\left[\begin{array}{l|l}
n & 2 \\
k & 2
\end{array}\right] /\left[\begin{array}{l}
n \\
k
\end{array}\right] \rightarrow e^{-y} .
$$

Киевский государственный университет Поступило им. Т.Г. Шевченко 09.08 .93 Исправленный вариант 14.04 .95

\section{СПИСОК ЦИТИРОВАННОЙ ЛИТЕРАТУРЫ}

[1] Эндрюс Г. Теория разбиений. М.: Наука, 1982. 